\title{
Determinantes de la deserción universitaria: un estudio de caso en la República Dominicana
}

Determinantes do abandono universitário: um estudo de caso na República Dominicana

Determinants of university dropout: a case study in the Dominican Republic

\author{
Rafael Marte Espinal \\ rmarte32@uasd.edu.do \\ Universidad Autónoma de Santo Domingo - República Dominicana \\ https://orcid.org/0000-0002-4302-4016
}

Lamec Fabián V

lf131519@gmail.com

Universidad Autónoma de Santo Domingo - República Dominicana

https://orcid.org/0000-0002-3144-0557

\begin{abstract}
RESUMEN
El objetivo de esta investigación es determinar los factores que motivan la deserción en la Universidad Autónoma de Santo Domingo, Recinto Santiago, periodo 2016-2019. Para dar respuesta a las preguntas y a los objetivos específicos de la misma, se llevó a cabo una investigación de campo, tipo descriptiva, con enfoque mixto), un diseño no experimental y de corte transversal. Para recoger la información se utilizó la técnica de la encuesta y la entrevista abierta. Se usó el muestreo probabilístico, aleatorio complejo en varias etapas y se extrajo una muestra de 415 estudiantes de una población de 12,716 discentes, de ambos sexos, de las distintas carreras que se imparten en este recinto y para la realización de los análisis estadísticos se utilizó el programa SPSS y Microsoft Excel. En general, los niveles de retiro o abandono de asignaturas son muy altos $85.6 \%$, así mismo los que retiran asignaturas, en su mayoría no se realizan de manera formal 59.04\%, el 75.3\% ha retirado entre 1 y 4 veces alguna asignatura. Las que tienen mayor nivel de retiro o abandono son: Educación y Medicina con $19 \%$ y $28 \%$ respectivamente. Por otra parte, el $15.8 \%$ de los encuestados tienen índice académico por debajo de 70 puntos, donde las carreras de Educación y Medicina tienen los menores índices representando el $16.3 \%$ y $32.6 \%$ respectivamente, el $44.2 \%$ de los que tienen índices por debajo de 70 puntos están en el rango de edad de 25 a 32 años, estos datos tampoco presentan ningún patrón predeterminado.
\end{abstract}

Palabras clave / Descriptores: Deserción universitaria. Deserción. UASD. Recinto Santiago.

\begin{abstract}
The objective of this research is to determine the factors that motivate desertion at the Autonomous University of Santo Domingo, Santiago Campus, period 2016-2019. To answer the questions and the specific objectives of the same, a descriptive field investigation was carried out, with a quantitative approach, a non-experimental and cross-sectional design. To collect the information, the survey technique and the open interview were used. The probabilistic, complex random sampling was used in several stages and a sample of 415 students was extracted from a population of 12,716 students, of both sexes, from the different careers that are taught in this campus and to carry out the statistical analyzes it was used the SPSS program and Microsoft Excel. In general, the levels of withdrawal or abandonment of subjects are very high $85.6 \%$, likewise those who withdraw subjects, most of them are not formally carried out $59.04 \%, 75.3 \%$ have withdrawn between 1 and 4 times a subject. Those with the highest level of withdrawal or abandonment are: Education and Medicine with $19 \%$ and $28 \%$ respectively. On the other hand, $15.8 \%$ of those surveyed have an academic index below 70 points, where Education and Medicine careers have the lowest indexes, representing $16.3 \%$ and $32.6 \%$ respectively, $44.2 \%$ of those with indexes below 70 points are in the age range of 25 to 32 years, these data do not present any predetermined pattern either.
\end{abstract}

Keywords: University dropout. Desertion. UASD. Santiago Campus

\section{RESUMO}

O objetivo desta pesquisa é determinar os fatores que motivam a deserção na Universidade Autônoma de Santo Domingo, Campus de Santiago, período 2016-2019. Para responder às questões e aos objetivos específicos das mesmas, foi realizada uma pesquisa de campo descritiva, com abordagem mista), de delineamento não experimental e transversal. Para a coleta de informações, utilizou-se a técnica de survey e a entrevista aberta. Foi utilizada amostragem probabilística aleatória complexa em várias etapas e extraída uma amostra de 415 alunos de uma população de 12.716 
alunos, de ambos os sexos, das diferentes carreiras ministradas neste campus, o programa SPSS e o Microsoft Excel. Em geral, os níveis de desistência ou abandono de disciplinas são muito elevados 85,6\%, assim como os que desistem de disciplinas, a maioria delas não são realizadas formalmente 59,04\%, 75,3\% desistiram entre 1 e 4 vezes uma disciplina. Aqueles com maior índice de desistência ou abandono são: Educação e Medicina com $19 \%$ e $28 \%$ respectivamente. Por outro lado, $15,8 \%$ dos pesquisados possuem índice acadêmico abaixo de 70 pontos, sendo que as carreiras de Educação e Medicina apresentam os menores índices, representando 16,3\% e 32,6\% respectivamente, 44,2\% daqueles com índices abaixo de 70 pontos estão na faixa etária na faixa de 25 a 32 anos, esses dados também não apresentam nenhum padrão pré-determinado.

Palavras-chave / Descritores: Abandono universitário. Deserção. UASD. Santiago Campus

\section{INTRODUCCIÓN}

El sistema de educación superior de la República Dominicana se ve afectado por el retiro permanente de los estudiantes, abandonando sus programas de clases, presentando altos niveles de deserción en las distintas universidades de la región y el país, esto afecta el tiempo de formación de los estudiantes en sus respectivas carreras.

El tema del presente artículo es la Deserción en la UASD Recinto Santiago, en los periodos 2016-2019, el cual es una dificultad que ocupa a las instituciones de nivel superior públicas y privadas. El objetivo de esta investigación es determinar las principales causas que inciden el retiro total o parcial de los estudiantes en la UASD Recinto Santiago, el centro de estudio es en el campus Universitario de la Barranquita, el cual consta de cinco edificios y el campo abierto (asignaturas sin asignar a algún docente).

Para llevar a cabo este propósito se hace necesario plantear los aspectos metodológicos, que involucran: planteamiento del problema, antecedentes, investigaciones locales e internacionales, estas contienen la revisión de la literatura existente más relevante a nivel nacional y de la región de Latinoamérica y el caribe, así como el diseño metodológico. Contemplando, además cuatro aspectos a trabajar, (1) Un Modelo Logístico Binario (MLB) para el caso de los desertores internos (2) Aplicación de la encuesta a estudiantes activos, (3) Aplicación de la encuesta a estudiantes retirados de forma definitiva y (4) Aplicación de la entrevista a los docentes activos.

Finalmente, se presentan las teorías de diversos autores sobre los factores y modelos de la deserción de los estudiantes en la educación superior, así como algunos resultados obtenidos de la aplicación de los instrumentos mencionados anteriormente y su interpretación.

\section{FUNDAMENTO TEÓRICO}

\subsection{La Deserción}

La deserción estudiantil universitaria es un fenómeno causal que tiene diferentes factores que confluyen, como aspectos de orden académico, institucional, personal, sociodemográfico (Barrero, 2015), en este sentido, la deserción se perfila como el abandono del proceso de formación académica de un individuo ya sea de forma voluntaria o no, o bien de forma definitiva o temporal en una institución de educación superior, debido a una serie de causas que convergen en aumentar la probabilidad de deserción del alumno.

La deserción es el proceso de abandono voluntario o forzoso de la carrera en la que se matricula un estudiante, por la influencia positiva o negativa de circunstancias internas o externas a él o ella (Steren y Arriaga , 2013), el autor resalta la diferencia entre rezago y deserción. El "rezago" es la prolongación de los estudios por sobre lo establecido formalmente para cada carrera o programa. 
De forma general la deserción universitaria es el abandono de los estudios en una institución de educación superior parcial o total debido a factores que afectan la continuidad del alumno en su proceso de formación. La deserción es el abandono prematuro de un programa de estudios antes de alcanzar el título o grado (Himmel, 2002), además considera el tiempo para retomar los estudios por parte del estudiante retirado.

\subsection{Factores asociados a la deserción Universitaria.}

Las causas de la deserción universitaria se deben a una serie de factores que afectan la permanencia del alumno en las aulas, sin embargo, no se han puesto de acuerdo en saber ¿cuáles factores pesan más a la hora del estudiante decidir si abandona o no las aulas?

Existen mayores posibilidades de que no ocurra la deserción si el alumno:

Primero, concluyen a tiempo sus estudios de bachillerato y con un buen rendimiento académico. Segundo, si en el examen de selección de ingreso a los estudios superiores logra un nivel adecuado de conocimientos en áreas como razonamiento verbal y español (Huesca \& Castaño, 2007). Sin embargo, los aspectos mencionados por los autores son solo académicos y la deserción al ser un fenómeno multicausal, implica una serie de factores que deben considerarse a saber, factores socioeconómicos, psicológicos, motivacionales, entre otros.

Otros autores clasifican los factores que afectan la estadía de los estudiantes en las aulas de las distintas universidades (Guzman, 2014), planteando la siguiente clasificación:

a) Abandono precoz: es el que ocurre cuando la persona, aun siendo aceptada por la universidad no se matricula.

b) Abandono temprano: se produce cuando el estudiante abandona sus estudios en el primer tramo de la carrera. Se incluye en esta categoría a los abandonos ocurridos en los primeros semestres de la carrera.

c) Abandono tardío: hace referencia al abandono del estudiante que ha superado al menos la mitad de los semestres establecidos en el programa académico.

El autor plantea, además, que estos tipos de abandono, el abandono tardío, es el "más costoso" (Guzman, 2014) para la universidad por los años invertidos en educación, sin lograr que el estudiante se titule. En los casos que la educación superior es financiada por el gobierno, como es el caso de República Dominicana, los costos de deserción resultan sumamente altos. En el caso específico de la República Dominicana los costos promedios por alumno que el gobierno realiza en la Universidad Autónoma de Santo Domingo (UASD), para el año 2003, es aproximadamente de US\$182.46 por estudiante matriculado en la UASD (Cabral, 2004)

Los factores que causan la deserción en tres grupos (García, 2014), Personales: que involucran las características personales de los estudiantes como la falta de actitud de logro en el crecimiento profesional. La incompatibilidad del tiempo dedicado al trabajo y a los estudios. Institucionales y pedagógicos: la deficiente orientación vocacional recibida antes de ingresar a la licenciatura, que provoca que los estudiantes se inscriban en las carreras profesionales sin sustentar su decisión en una sólida información sobre las mismas. Socioeconómicos y laborales: las condiciones económicas desfavorables del estudiante y la carencia de financiamiento.

Al estudiar los factores asociados a la deserción, encontramos una serie de elementos que colaboran para que entre en vigencia la decisión del estudiante de retirarse de forma parcial o definitiva de las aulas universitaria, tales factores, personales, institucionales y pedagógicos así 
como socioeconómicos y laborales, en tal sentido, la deserción puede ocurrir en cualquier momento de la vida escolar, al inicio, durante los primeros semestres de carrera o al final del programa o carrera cursado (González, 2005). Por las razones antes referidas, Se propone un modelo ideado por Bean, \& Metzner, (1985), para conceptualizar el síndrome de la deserción, basado en los factores ya mencionados en la presente investigación

\subsection{Investigaciones Nacionales.}

En la República Dominicana se consideran tres estudios importantes sobre la deserción universitaria en los últimos anos, a saber:

El estudio Deserción Académica en la UASD determinó que "alrededor del 75\% de los estudiantes que ingresaron a la universidad a mediados de la década de los 90 , abandonaron sus aulas, así mismo el estudio, al mismo tiempo pone de manifiesto el alto índice de deserción y baja eficiencia de titulación encontrados en la universidad estatal en los años 1999-2003”. (Cabral, M. 2004)

Descensión universitaria en la Universidad Abierta para Adultos (UAPA), esta institución es de carácter virtual y/o semipresencial, las conclusiones de Jiminián $(2009$, p. 82) en su trabajo son: los alumnos que residen en zonas rurales alcanzan mayor tasa de deserción. Los alumnos que tienen como último grado el de bachiller la tasa de deserción alcanza hasta el 53\%. Respecto a la procedencia de los estudios secundarios, los de instituciones semiprivadas tienen la menor tasa de deserción, seguidos de los que vienen de instituciones públicas, los de instituciones privadas presenta la mayor tasa de deserción. Jiminián (2009) construye un perfil sociodemográfico para los alumnos desertores de UAPA, a saber, masculino, soltero, sin hijos, de edad entre los 21-35 años, su último grado al ingresar a la UAPA es bachiller, tienen de 5-10 años de haber cursado el mismo, residen en zona rural, proceden de instituciones privadas, no trabajan y de los que trabajan desertan más aquellos que perciben de US\$139.00-US\$278 dólares.

La deserción y permanencia de los estudiantes en el sistema público y privado de la Republica Dominicana, concluye que "A nivel general, tanto en instituciones públicas como privadas, la tasa de deserción sobrepasa el 50\%, dentro de esta alta tasa de deserción global, la deserción en los hombres sigue siendo mayor que en las mujeres" (Hernández, 2012, p21)., lo cual constituye una problemática general en las instituciones de educación superior.

\subsection{Investigaciones Internacionales}

Algunas investigaciones que se pueden citar en la región de América Latina sobre deserción universitaria son las siguientes:

Una recopilación de estudios sobre Repitencia y Deserción universitaria en América Latina (Guzmán, 2014), para el caso de Bolivia, puntualiza que la deserción más allá de ser un indicador de los niveles de eficiencia de las instituciones, tiene implicancias en tres planos importantes: en el plano personal de quienes abandonan sus estudios, en el plano social y en el plano institucional. Mientras en una investigación realizada en Guatemala en la Universidad de San Carlos para la carrera de Medicina (Guzmán, 2014, p.14), este estudio concluye que los factores que se predisponen a la deserción estudiantil en el primer año de la carrera de Medicina se agrupan en 2 aspectos: académicos y no académicos. Los académicos: entre los que se incluye la no aprobación de los siguientes cursos: Química, Física, Biología y Psicología. Mientras que los no académicos: que incluyen el hecho de no ser cristiano, el que los padres no sean universitario y que el estudiante debe efectuar un trabajo remunerado simultáneamente con sus estudios. 
En una investigación referentes a los factores incidentes en el abandono escolar en América Latina se establece que los numerosos y diversos estudios que dan cuenta de factores que inciden en el desempeño y trayectoria escolar y que analizan especialmente sus efectos e impactos sobre los principales indicadores de fracaso escolar se caracterizan por dos orientaciones teóricas diferentes que buscan identificar y comprender el origen y dinámica que genera y posibilita bajos aprendizajes, reprobación de grados, ausencias reiteradas, desmotivación por avanzar y aprender, todos signos evidentes de un proceso de fracaso escolar, cuya último eslabón es el abandono y la desvinculación definitiva de la escuela (deserción) y del sistema (Román, 2013, p. 37)

Otra investigación analizada es un estudio realizado en La Universidad de Costa Rica, el cual concluye que una de las principales causas de la deserción, que el estudiantado no logra ingresar a la carrera que desean seguir, situación que se ratifica en que un $63.4 \%$ al salir de la UCR, lo hace en el momento en que está empadronado en carreras que no eran las que quería estudiar cuando ingresó (Rodríguez \& Sánchez V, 2005, p. 14)

\section{PROCEDIMIENTOS METODOLOGICOS}

En esta sección del presente artículo, se describe la metodología utilizada, así como la población objeto, descripción general de las variables, así como el alcance y las limitaciones. Este trabajo es de carácter descriptivo, exploratorio y correlacional, con un enfoque cuantitativo y cualitativo.

Es exploratorio ya que el objetivo es examinar un tema o problema de investigación poco estudiado, o no ha sido abordado antes (Sampieri, Fernández \& Baptista, 2006, p.26), en este sentido las causas que provocan el fenómeno de la deserción no se han investigado en la UASD Recinto Santiago. Por otra parte, es descriptivo, ya que un estudio descriptivo nos permite decir ¿Cómo es? y ¿Cómo se manifiesta? determinado fenómeno. Los estudios descriptivos buscan especificar las propiedades importantes de personas, grupos, comunidades o cualquier otro fenómeno que sea sometido a análisis, lo cual permite especificar las causas que generan la deserción académica en la UASD Recinto Santiago.

Es correlacional ya que la utilidad y el propósito principal de los estudios correlacionales son, saber cómo se puede comportar un concepto o variable conociendo el comportamiento de otra u otras variables relacionadas (Martínez, 2006, p.128), de esta forma podemos predecir el comportamiento del estudiante que tenga características propias de un desertor.

Es de enfoque mixto, puesto que posee características del enfoque cuantitativo, específicamente la aplicación del cuestionario estructurado para determinar los factores de la deserción y se utilizan modelos matemáticos para analizar los factores y hallazgos de fuentes cuantitativas. Del mismo modo, se aplica el método cualitativo, ya que se trata de identificar la naturaleza profunda de las realidades, su estructura dinámica, aquella que da razón plena de su comportamiento y manifestaciones. En este sentido, los factores a analizar son: sociales, económicos, académicos y personales, además el enfoque es cuantitativo debido puesto que se cuantifican las respuestas, además se estima la probabilidad de desertar del estudiante de una cohorte de 2016-2019 de la UASD Recinto Santiago.

Debido al tipo de estudiante que está inscrito en la UASD recinto Santiago, este trabajo tiene dos enfoques a analizar: (1) Estudiantes inscritos y activos que desertan, abandonan o retiran asignaturas, aunque se mantienen activos en la universidad, estos son potenciales desertores definitivos de la universidad y (2) Los estudiantes que se retiran de forma definitiva o temporal de la institución. 


\subsection{Modelo Logístico Binario (MLB)}

En el primer caso se analiza el estudiante que abandona o retira una o más asignaturas durante uno o más semestres, en este caso se propone un Modelo Logístico Binario para tratar de predecir el impacto de algunas variables cuantitativas y cualitativas en la decisión de retirar o abandonar asignaturas y que posteriormente puede conllevar a un retiro definitivo de la institución. La variable a analiza es retiro de asignatura, es una variable dicotómica que representa la condición de retiro o abandono de la signatura por parte de un estudiante, donde 1 representa retiro o abandono de la asignatura y 0 no retiro o abandono. La variable de retiro de la asignatura está asociada a seis variables explicativas, una numérica y cinco dicotómicas.

La regla de decisión sobre el rechazo o no del modelo se basará en el contraste de p-valor de los estadísticos de pruebas a un nivel de significación estadística de 0.10 (10\%), Dada la naturaleza dicotómica de la variable dependiente, se propone un modelo de regresión logística binario, para estimar la probabilidad de que un estudiante deserte (Retire asignaturas o las abandone).

$$
P_{i}=\frac{1}{1+e^{-z}} \text { donde } z=\beta_{0}+\beta_{1} x_{1}+\beta_{2} x_{2}+\cdots+\beta_{k} x_{k} \text { o bien } \operatorname{Ln}(z)=\beta_{0}+\sum_{k=1}^{n} \beta_{k} x_{k}
$$

Donde $\mathrm{Pi}$ es la probabilidad de desertar (retiro o abandono de asignaturas) y $\mathrm{e} \approx$ $2.71828182845905 \ldots$

El modelo no es lineal en los parámetros por lo cual no se pueden hacer estimaciones de mínimos cuadrados ordinarios (MCO), así mismo, el supuesto de homocedasticidad de la varianza no se cumple en los modelos de regresión de variables explicadas binarias debido a que la varianza depende de los valores que suma Pi. Para corregir esta violación del supuesto homocedasticidad de los términos de error aleatorios se ponderan por

$$
\sqrt{w_{i}}=\sqrt{N_{i} \widehat{P}_{i}\left(1-\widehat{P}_{i}\right)}
$$

Considerando que para muestras grandes el factor de ponderación tiene un efecto de corrección pequeño. Por ejemplo, se espera que los coeficientes de X3, X4, X2, X5 y X6, sean positivos, es decir, aumenten los odds ratio, la razón de probabilidades en favor de encontrar un estudiante que deserte respecto de la probabilidad de que no deserte. De forma general, para cada uno de estos coeficientes se espera que la hipótesis nula $\mathrm{H} 0$ sea $\beta \mathrm{i}>0$. Por otra parte, se espera que el coeficiente de X1, sea negativo, es decir, disminuyan los odds ratio. De forma técnica, H0 sea $\beta \mathrm{i}$ $<0$.

En cuanto al ajuste del modelo, el contraste sobre los parámetros utilizará el test de Wald que sigue un modelo de chi cuadrado $\left(\mathrm{N}^{2}\right)$, se basa en la normalidad asintótica de los coeficientes del modelo. Donde H0: $\beta \mathrm{i}=0$, mientras que $\mathrm{H} 1$ expone que al menos uno de los $\beta \mathrm{i}$ es distinto de cero.

$$
w=\frac{\left(b_{j}-\beta_{0}\right)^{2}}{\operatorname{Var}\left(b_{j}\right)} \rightarrow \mathrm{N}^{2}
$$

Para la interpretación de los coeficientes, en el modelo, tenemos que, si el coeficiente $\beta \mathrm{i}>0$ indica que la variable genera un incremento en la probabilidad del evento. En el caso de que el coeficiente $\beta \mathrm{i}<0$ indica que la variable genera una disminución de la probabilidad del evento, finalmente, si el coeficiente $\beta \mathrm{i}=0$, no genera cambio en la probabilidad de ocurrencia del evento. 
Para analizar los residuos se utilizará la devianza, devianza pequeñas significa un buen ajuste del modelo, devianza grandes significa un mal ajuste del modelo. Esto no es más que, el doble logaritmo del estadístico de verosimilitud el cual tiene una distribución chi cuadrado.

$\mathbb{N}^{2}=2 L L_{\text {nuevo }}-2 L L_{\text {referemeial }}$

Los grados de libertad son $g l=k_{\text {mwevo }}-1$

Finalmente se evaluará el ajuste general del modelo mediante el pseudo R2 de McFadden.

$$
R^{2}=1-\frac{\hat{L}_{\text {modelo }}}{\tilde{L}_{\text {intercepto }}}
$$

Este estadístico compara la log-verosimilitud del modelo como suma de errores del modelo entre el log-verosimilitud del modelo que solo tiene el término del intercepto como suma de cuadrados totales.

La investigación es de campo, donde se aplicaron los instrumentos (cuestionarios) a los estudiantes inscritos activos y a los estudiantes desertores, mediante esta aplicación se generaron dos bases de datos, una de estudiantes inscritos y otra de estudiantes retirados en el período 20162019 en la UASD Recinto Santiago. La gestión de las bases de datos se realizó mediante Microsoft Excel e IBM SPSS. La presentación de los datos se hizo en Microsoft Excel e IBM SPSS.

Tabla No. 1.

Operacionalización de las variables del Modelo Logístico Binario (MLB), definición conceptual y operacional.

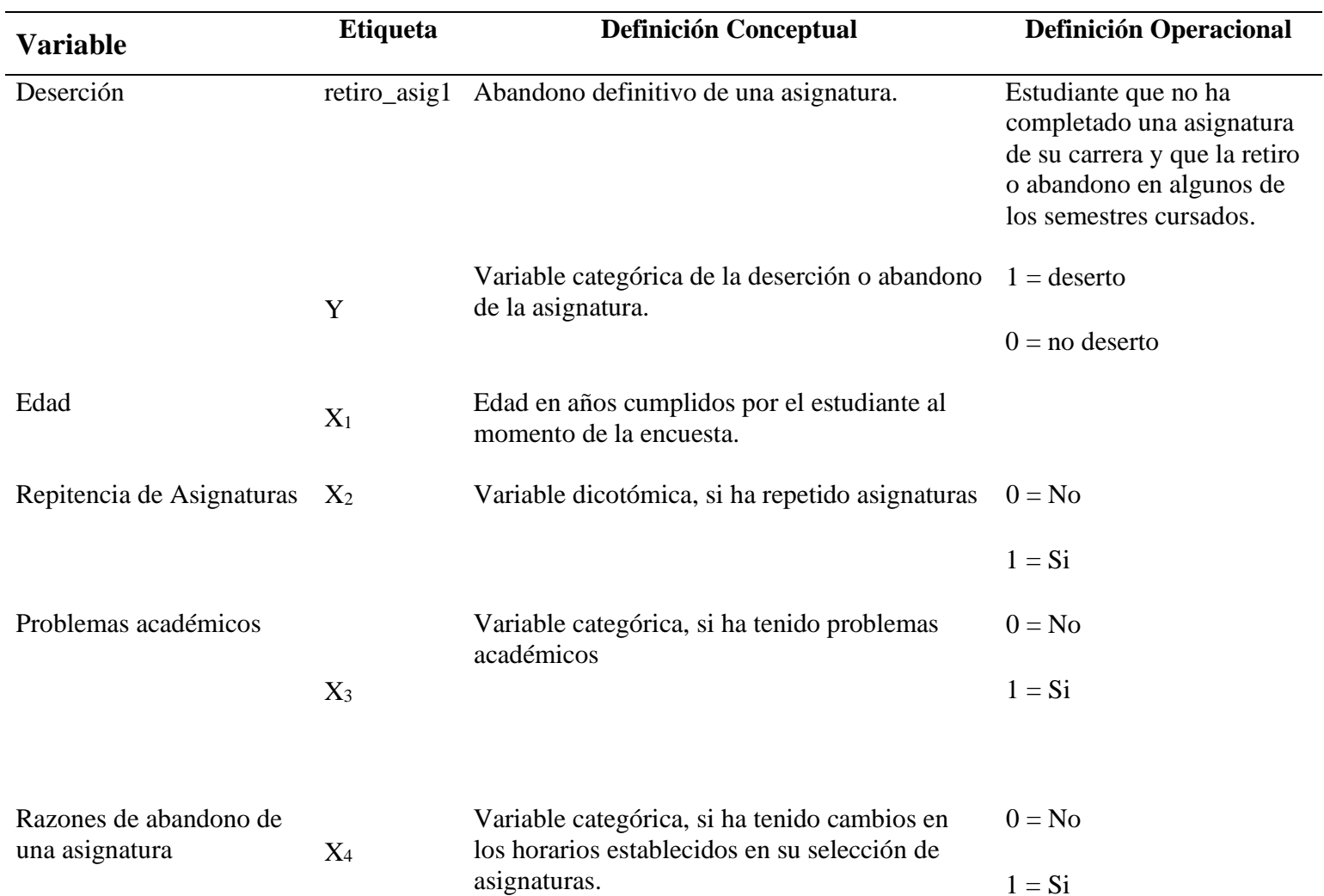


Razones de abandono de una asignatura una asignatura
Variable categórica, si ha tenido problemas con $0=$ No

la metodología de trabajo del docente.

$1=\mathrm{Si}$

$0=$ No

Variable categórica, si ha tenido cambio de

carrera o programa de formación.

$1=\mathrm{Si}$

Fuente: elaboración propia

\subsection{Deserción Interna (Abandono o Retiro de Asignaturas)}

La encuesta sobre los factores y causas que dificultan la permanencia de los estudiantes inscritos y activos de la UASD Reciento Santiago, es de carácter cualitativo y tiene la finalidad de conocer el nivel de dificultad que representan factores específicos que miden niveles de deserción ya definidos en la región de América Latina y el Caribe. Como objetivo principal se pretende describir el nivel de dificultad que representan estos factores específicos que motivan la deserción en los estudiantes de la UASD Recinto Santiago, de forma más precisa se pretende:

1. Comparar el nivel de dificultad de los factores específicos de deserción en los estudiantes, segregados por sexo, edad y carreras.

2. Identificar las competencias directas de la universidad y del cuerpo docente para nuevas políticas de mejora y reducción de la deserción.

3. Promover investigaciones posteriores que aborden a los estudiantes que han desertado y que permitan analizar de forma más minuciosa el abandono por carreras de forma particular.

\subsection{Marco Muestral, Población y Muestra}

El marco muestral se conformó de la siguiente forma: las secciones programadas para el período 2019-20 conforman la unidad muestreo (UM1) para identificar factores y causas que se presentan como dificultades en los estudiantes matriculados y activos de la UASD Recinto Santiago, dado el enfoque del estudio, los estudiantes conforman la unidad primaria de análisis (UPA2), que son los posibles desertores definitivos, considerando los modelos de los factores y causas específicas de deserción.

Se realizó un muestreo complejo en varias etapas, en el cual las unidades muestrales fueron seleccionadas por muestreo aleatorio simple:

1- Etapa 1: Se realizó un conteo de los edificios (UPM), siete en total, de los cuales se seleccionaron cuatro al azar.

2- Etapa 2: Se realizó un conteo de los niveles de cada edificio, doce en total de los cuales se seleccionaron ocho al azar.

3- Etapa 3: Se realizó un conteo de las aulas dentro de cada nivel, 76 en total, de las cuales se seleccionaron 28 de forma aleatoria.

4- Etapa 4: Se selecciono al azar el estudiante a encuestar (UPA).

No se realizaron las entrevistas por carreras ya que en la mayoría de las secciones podemos encontrar estudiantes de diversas carreras, en este sentido, la clasificación no se realizó, sino que se

\footnotetext{
${ }^{1}$ Unidad de Muestreo (UM): Se refiere al elemento de la población objeto de estudio, el cual va a ser seleccionado de forma aleatoria a la cual cada miembro de la población debe pertenecer a una y solo una unidad de muestreo

2 Unidad Primaria de Análisis (UPA): Es la unidad sobre la cual se va a realizar la investigación, es decir, los individuos a investigar.
} 
seleccionaron las secciones de docencia en las aulas seleccionadas en la etapa 3. Para los estudiantes matriculados correspondientes al semestre 2019-20, fueron abordados en los horarios siguientes: matutino de 7:00 a 12:00 m, vespertino de 1:00 pm a 6:00 pm y nocturno 6:00 a 10:00 pm. Además, se solicitó la ayuda de los profesores para permitir la intervención de los encuestadores, se realizaron con los estudiantes aclaraciones básicas del llenado del instrumento, haciendo énfasis en la sinceridad e individualidad de las respuestas y el anonimato de las mismas.

La población, para hacer esta investigación se considera el total de los estudiantes de la UASD Recinto Santiago, los cuales ascienden a 12,716 alumnos en promedio, inscritos y activos hasta ese momento, según los datos del departamento de registro, estos datos fueron obtenidos a través del sistema de registro y proceso de estudiantes. Las unidades de muestreo seleccionadas fueron las secciones programadas correspondientes a UASD Recinto Santiago para el periodo 2019-20, las cursan los estudiantes inscritos y activos en el mismo periodo en vigencia, La unidad primaria de análisis la conforman los estudiantes matriculados y activos, en el mismo periodo.

Para el cálculo del tamaño de la muestra se utilizó la fórmula de Fisher-Navarro

$n=\frac{z^{2} \cdot n \cdot p(1-p)}{e^{2}(n-1)+z^{2} \cdot p(1-p)}, \quad n=373$

Obteniendo una muestra de 373, estudiantes activos, que aplicándole el factor de expansión post muestreo del $10 \%$.

$$
\begin{aligned}
& n=\frac{z^{2} \cdot N \cdot p(1-p)}{e^{2}(N-1)+z^{2} \cdot p(1-p)} \times \frac{1}{1-f \cdot \operatorname{Exp} .} \\
& n=373 \times \frac{1}{1-0.10}=415
\end{aligned}
$$

\subsection{Aplicación de la encuesta a estudiantes retirados de forma definitiva.}

La encuesta sobre los factores que causaron la no permanencia de los estudiantes en la UASD Recinto Santiago en el periodo en estudio es de carácter cualitativo y tiene la finalidad de conocer los distintos factores que motivaron la deserción definitiva de su programa de estudio. Como objetivo principal se pretende determinar los distintos factores que motivaron la deserción, de forma más puntual se pretende: comparar el nivel de dificultad de los factores específicos de deserción en los estudiantes, segregados por sexo, edad y carreras. Promover investigaciones posteriores que aborden a los estudiantes que han desertado y que permitan comparar y validar los resultados de esta.

Debido a las dificultades presentadas para poder acceder las unidades primarias de análisis, nos vemos en la necesidad de realizar un marco muestral de unidades disponibles, es decir, acceder a las UPA, dentro de las posibilidades con las que contamos. Se visitaron las distintas universidades que se encuentran en la provincia de Santiago y se entrevistaron algunos estudiantes retirados vía telefónica, en otros casos se llevaron a cabo entrevistas en algunos barrios, contactando algunos desertores. Finalmente, la muestra disponible fue de 86 desertores. 


\subsection{Aplicación de la entrevista a los docentes activos.}

En esta parte del estudio de la deserción se abordaron a expertos (maestros activos) y como método de recolección de información se programaron entrevistas. Se elaboró un cuestionario de preguntas abiertas, con la finalidad de que el tema se desarrolle de manera óptima y que se manifieste la posición de la parte académica con respecto a la repitencia y la deserción. Esta entrevista tiene la finalidad visualizar el nivel de información que tienen los directores de departamentos y docentes acerca de los fenómenos de repitencia y deserción en UASD Recinto Santiago. En ese sentido se pretende: primero, comparar la información dada por los profesores con los resultados del estudio de campo de los estudiantes inscritos y activos. Segundo, conocer las medidas y las políticas aplicadas desde sus competencias como docentes en función. Tercero, conocer las medidas y las políticas propuestas desde sus competencias como docentes, en función.

La entrevista fue aplicada a los maestros activos residentes en UASD Recinto Santiago, mediante un dialogo directo con los interesados y se acordó, de forma verbal el consentimiento y una cita para la entrevista, previa aclaración del tema, posteriormente se recibió la confirmación de cada docente, vía mensaje de texto y/o correo electrónico (tanto de la cita cómo del consentimiento), se enumeran las preguntas (abiertas) a realizar en horario adecuado que no afecte su labor de docencia.

\section{RESULTADOS Y DISCUSIÓN}

\subsection{Caso 1: Modelo Logístico Binario.}

El Modelo Logístico Binario creado para la deserción interna en la UASD Recinto Santiago, para la variable retiro de asignaturas de su carga académica, queda explicado por las variables Sector_P (X1), rep_asignaturas (X2), pro_académicos (X3), razones de abandono, que contemplan los aspectos de: cambios de horarios (razonesz_abandono_1 = X4), metodología usada por el docente (razones_abandono_3 = X5), cambios de carreras por parte de los estudiantes (razones_abandono_4 = X6) y edad en años cumplidos $($ edadDIC $=$ X7), en ese mismo sentido, el modelo tiene un poder explicativo de un $89.7 \%$ con un punto de corte del $50 \%$ (0.50), además, el modelo se ejecutó con una confianza del $90 \%$ y una significancia del 10\% (0.10).

\subsection{Resumen del Modelo}

En el resume del modelo se interpretan los resultados de los signos de los coeficientes, en este sentido se obtiene:

1 - $\mathrm{X}_{1}=1.438, \mathrm{Si}$ los estudiantes provienen del sector público aumenta la probabilidad de deserción.

2- $\mathrm{X}_{2}=2.123, \mathrm{Si}$ el estudiante ha reprobado asignatura antes aumenta la probabilidad de reprobar asignaturas.

3- $\mathrm{X}_{3}=-1.034$, cuando no hay problemas académicos, disminuye la probabilidad de deserción.

4- $\mathrm{X}_{4}=-2.254$, cuando no hay cambio de horario, la probabilidad de deserción disminuye.

5 - $\mathrm{X}_{5}=-1.292$, cuando no hay problemas con la metodología del docente, disminuye la probabilidad de abandono.

6- $\mathrm{X}_{6}=-1.595$, si no hay cambio de carreras, la probabilidad de abandono de las asignaturas disminuye.

7- $\mathrm{X}_{7}=1.608$, a mayor edad, mayor es la probabilidad de deserción o abandono.

8- $\beta_{0}=10.352$, en presencia de las demás variables la probabilidad de deserción o abandono de asignaturas aumenta. 


\subsection{Interpretación de los coeficientes transformados.}

En el caso de los coeficientes transformados, se identifica cuantas veces se afecta la probabilidad de ocurrencia o no de la deserción.

1- $\operatorname{Exp}\left(\beta_{1}\right)=4.211$, cuando el estudiante es del sector público, la probabilidad de abandono es 4.211 veces mayor.

2- $\operatorname{Exp}\left(\beta_{2}\right)=8.353$, cuando el estudiante tiene historia de repitencia de asignaturas, la probabilidad de abandono de las asignaturas aumenta en 8.353 veces más.

3- $\operatorname{Exp}\left(\beta_{3}\right)=0.356$, cuando el estudiante no tiene problemas académicos, la probabilidad disminuye 0.356 veces.

4- $\operatorname{Exp}\left(\beta_{4}\right)=0.105$, cuando el estudiante no tiene cambio de horarios, la probabilidad de abandono disminuye 0.105 veces.

5- $\operatorname{Exp}\left(\beta_{5}\right)=0.275$, cuando no hay problemas con la metodología del docente disminuye la probabilidad de abandono 0.275 veces.

6- $\operatorname{Exp}\left(\beta_{6}\right)=0.203$, si no hay cambios de carreras, la probabilidad de abandono de las asignaturas disminuye 0.203 veces.

7- $\operatorname{Exp}\left(\beta_{7}\right)=4.992$, a mayor edad, la deserción o abandono aumenta 5 veces, aproximadamente.

8- $\operatorname{Exp}\left(\beta_{0}\right)=31,315.348$, en presencia de las demás variables la probabilidad de abandono de asignaturas es 31,315 veces más aproximadamente.

EL Modelo: $Z=\beta_{0}+\beta_{1} * X_{1}+\beta_{2} * X_{2}+\beta_{4} * X_{4}+\beta_{5} * X_{5}+\beta_{6} * X_{6}+\beta_{7} * X_{7}+\varepsilon_{i}$

$$
Z=10.352+1.438 * X_{1}+2.123 * X_{2}-1.034 * X_{3}-2.254 * X_{4}-1.292 * X_{5}-1.595 * X \_6+1.608 * X_{7}
$$

$P(Y)=\frac{1}{1+e^{-2}}$

La bondad de ajuste es más efectiva en la opción de SI, para la clasificación de los desertores internos, ya que los valores observados y esperados son muy similares, en el caso de la opción NO, las primeras iteraciones son parecidas, variando de forma significativas en las iteraciones posteriores, esto implica una precisión mayor en clasificar cuando el desertor interno ha retirado o abandonado materias, estos datos se reflejan en la tabla de clasificación, es decir, los porcentajes correctos nos dicen que los que no retiraron materias se clasificaron correctamente $43.6 \%$ y los que si retiraron el $97.4 \%$.

\subsection{Caso 2: Deserción Interna.}

Los niveles de retiro o abandono de asignaturas son muy altos $85.6 \%$, así mismo los que retiran asignaturas, en su mayoría no se realizan de manera formal 59.04\%. El $75.3 \%$ ha retirado entre 1 y 4 veces alguna asignatura. El estado civil de casados aumento aproximadamente en un $18 \%$, de igual forma, la cantidad de encuestados con hijos, aumento en 19\% aproximadamente. En cuanto a las carreras, las que tienen mayor nivel de retiro o abandono son Educación y Medicina con $19 \%$ y $28 \%$ respectivamente, además, estas últimas carreras entienden que las razones planteadas son motivo suficiente para retirar o abandonar las asignaturas. 
Tabla No. 2

Razones de retiro o abandono por carrera, UASD Recinto Santiago 2016-2019

\begin{tabular}{|c|c|c|c|c|c|c|c|}
\hline Razón & Pedagogía & $\begin{array}{l}\text { Ed. } \\
\text { Física }\end{array}$ & Medicina & $\begin{array}{l}\text { Adm. } \\
\text { Empresas }\end{array}$ & Ingenierías & Mercadeo & Psicología \\
\hline $\begin{array}{l}\text { Cambio de } \\
\text { Horario }\end{array}$ & 13.50 & 12.90 & 31.80 & 3.00 & 6.10 & 2.30 & 3.80 \\
\hline $\begin{array}{l}\text { Metodología del } \\
\text { Maestro }\end{array}$ & 12.40 & 7.30 & 27.70 & 10.20 & 9.50 & 5.10 & 5.10 \\
\hline $\begin{array}{l}\text { Cambio de } \\
\text { Carrera }\end{array}$ & 23.50 & 15.80 & 10.50 & 5.30 & 2.60 & 10.50 & 7.90 \\
\hline $\begin{array}{l}\text { Choque en } \\
\text { Horario de } \\
\text { Trabajo }\end{array}$ & 22.30 & 12.30 & 25.40 & 7.70 & 3.10 & 6.90 & 1.50 \\
\hline
\end{tabular}

Fuente: elaboración propia

\subsection{Caso 3: Estudiantes Retirados.}

Las razones de abandono por parte de los estudiantes, se resaltan las razones económicas y las razones académicas, con 49 (57\%) y 48 (55.8\%). Las medidas que los desertores entienden colaboran más con la disminución de la deserción, son mejorar los horarios de clases (66.3\%), así como realizar programas de motivación $(48.8 \%)$, medidas que son planteadas por los docentes en sus sugerencias.

En cuanto a los factores que, a juicio de los desertores, motivan la deserción en la UASD Recinto Santiago, el $62.8 \%$ entiende que las condiciones económicas desfavorables son un factor importante, el $64 \%$ entiende que la metodología usada por el maestro motiva al retiro, el $80.2 \%$, que la mala preparación en los niveles preuniversitario es fundamental en la deserción, por último, la mala elección de la carrera universitaria representa el 51.2\% de los entrevistados.

Tabla No.3

Factores que motivan la deserción interna. Desertores 2016-2019

\begin{tabular}{|c|c|c|c|}
\hline Factores & Si & No & No sabe \\
\hline Condiciones económicas desfavorables & 62.80 & 20.90 & 16.30 \\
\hline $\begin{array}{l}\text { Métodos de evaluación usados por la } \\
\text { universidad }\end{array}$ & 66.30 & 22.10 & 11.60 \\
\hline $\begin{array}{l}\text { Falta de preparación académica de los } \\
\text { docentes }\end{array}$ & 50.00 & 32.60 & 17.40 \\
\hline Metodología usada por los docentes & 64.00 & 16.30 & 19.60 \\
\hline $\begin{array}{l}\text { Deficiencia de los estudiantes por la mala } \\
\text { preparación en la educación preuniversitaria }\end{array}$ & 80.20 & 16.30 & 3.50 \\
\hline Falta de ayuda por parte de la universidad & 58.10 & 37.20 & 4.70 \\
\hline $\begin{array}{l}\text { Mala elección de la carrera por parte de los } \\
\text { estudiantes }\end{array}$ & 51.20 & 34.90 & 14.00 \\
\hline
\end{tabular}

Fuente: elaboración propia

4.6 Caso 4: Entrevista a los docentes activos de la UASD Recinto Santiago. 
En cuanto al fenómeno de la deserción la mayoría de los docentes coinciden en que es multicausal y multifactorial y además no es una problemática exclusiva de la UASD Recinto Santiago, sino que abarca a la UASD en todo su contexto y otras instituciones de educación superior, plantean algunos de los factores que, a su juicio, son fundamentales en el proceso de deserción, como son: empleo, faltad de recursos económicos y mala distribución de los horarios de docencia, planteando a posibilidad de que la universidad articule las carreras en bloques, con la finalidad de ayudar al avance de los estudiantes.

Entre las medidas que los docentes sugieren para ayudar a contrarrestar la deserción, tenemos: Motivación y orientación, estas son medidas que resaltan en el análisis de los resultados de los alumnos desertores, dar seguimiento a las inquietudes de los alumnos y usar recursos tecnológicos, entre otras. Para el caso de las medidas tomadas por el recinto para disminuir la deserción, pocos son los docentes que tienen conocimiento de medidas que se estén tomando para trabajar en la disminución de la deserción en la UASD Recinto Santiago, solo se mencionó la gestión de carreras técnicas y la dinamización del quehacer académico.

Las principales sugerencias que los maestros dan para disminuir la deserción son: puntualizar la oferta académica en base a la necesidad de la región, mejorar el trato a los alumnos y ofrecer talleres de pedagogía a los docentes, ofrecer nuevas carreras en la oferta curricular, ofrecer carreras técnicas, mejorar el transporte hacia la universidad ya que se encuentra en una zona alejada de la mayoría de los barrios y pueblos de la provincia, aplicar pruebas de aptitudes serias, esto, para mejorar las orientaciones académicas a los estudiantes, investigar causas socioeconómicas que causan la deserción y tomar decisiones en función de estas, entre otras.

\section{CONCLUSIÓN}

El presente artículo tiene como objetivo determinar los factores que influyen en la deserción de los estudiantes de la Universidad Autónoma de Santo Domingo (UASD) en el periodo 20162019. En ese orden, se encuestaron los estudiantes activos de distintas carreras los cuales resaltaron las siguientes razones como motivos de deserción: primero, cambio de horario, siendo medicina la carrera con mayor nivel de deserción (31.80\%), considerar que este nivel de deserción puede estar influenciado por que esta carrera tiene un alta matricula; seguida por pedagogía (13.50\%) y Educación Física (12.90\%). Segundo, la metodología usada por los docentes influye también en la deserción, puesto que medicina tienen $27.70 \%$ de deserción por esta razón. Tercero, el cambio de carrera es otra razón influyente donde pedagogía tiene $23.50 \%$ en la proporción de desertores. Cuarto, el choque con horarios de trabajo o laboral influye asimismo de forma significativa, en este caso la carrera de medicina tiene $25.40 \%$ de nivel de deserción y pedagogía con $22.30 \%$, porcentajes considerables y altos. Mientras que las principales limitantes que se presentaron fue el acceso a los estudiantes que ya se habían retirado de las asignaturas y de la universidad, la construcción de este marco muestral considero el rediseño total del muestreo de esta parte del estudio.

La fortaleza de esta investigación radica en que se fundamenta en el enfoque mixto, puesto que en este caso permite determinar de forma cualitativa y cuantitativa los factores determinantes en la deserción universitaria. También, los cuestionarios estructurados son de vital importancia en la recolección de los datos con los estudiantes inscritos y retirados. Así mismo, la entrevista con preguntas abiertas permite abordar el tema en forma amplia por expertos en el tema, y sin limitaciones en el planteamiento de su parecer respecto a la deserción y las medidas que deben tomarse para manejar el flagelo. Sin embargo, se presentaron limitaciones para la recolección de los datos, tanto con los estudiantes como con los docentes. En el primer caso, acceder a los estudiantes retirados de la universidad fue un reto, ya que los mismos cambian de dirección y teléfono con frecuencia. Mientras que, para el caso de los maestros, al tener que abordarlos en horario de trabajo, fue necesario presentarse en varias ocasiones para concertar la entrevista, en algunos casos hasta 
fuera del recinto universitario donde se realizó el estudio. En el caso de este estudio las anteriores referidas fueron las principales limitantes en la recolección de los datos y aplicación de la metodología del estudio.

$\mathrm{Al}$ abordar los estudiantes sobre los factores que los motivaron a retirarse de sus respectivas carreras se encontraron factores propios de la situación misma de los alumnos que influyen en la deserción, así como factores que son intrínsecos de la institución, dentro de estos las condiciones económicas del alumnado $(62.80 \%)$ un porcentaje bastante elevado y que podría servir de referencia para futuras investigaciones, la mala elección de las carreras por parte de los estudiantes $(51.20 \%)$ y deficiencia en la preparación preuniversitaria $(80.20 \%)$ porcentaje que resulta muy elevado y que debería indagarse más en estudios venideros, así mismo los métodos de evaluación de la universidad $(66.30 \%)$ y falta de ayuda por parte de la institución (58.10\%).

Finalmente, se recomienda abordar nuevos tópicos de investigación tales como, deserción por carreras, deserción por sector educativo de procedencia, deserción en su primer año de carrera y en años posteriores al primer año de carrera, entre otros, así como estudiar otros escenarios que involucren la deserción tales como las escuelas, facultades y otras instituciones de educación superior. Además, la preparación preuniversitaria como factor fundamental en la deserción universitaria, ayudando a mejorar la calidad educativa del país y disminuya de forma eficiente el fenómeno de la deserción

\section{REFERENCIAS}

Barrero, F. (2015). Investigación en deserción estudiantil universitaria. (Revista Educación y Desarrollo Social), 86101.

Bean, J. P., \& Metzner, B. S. (1985). A conceptual model of nontraditional undergraduate student attrition. Review of educational Research, 55(4), 485-540.

Steren, B. Arriaga, J. (2013). Una Vision Integral del Abandono, EdiPUCRS, Porto Alegre - RS - Brasil

Cabral, M. (2004). Desercion Universitaria en la República Dominicana. Estudio Para La IESALC/UNESCO 2000 2005.

García, M. (2014). Rendimiento académico y abandono universitario: Modelos, resultados y alcances de la producción académica en Argentina. (Revista Argentina de Educación Superior, (8), 9-38).

González, L. (2005). Repitencia y deserción universitaria en América Latina. Informe Sobre La Educación Superior En América Latina y El Caribe 2000-2005, 156-168.

Guzman, D. (2014). Factores predisponentes a la deserción en primer año de la carrera de Medicina, Guatemala.

Hernandez, W. (2012). Permanencia , y estrategias de tutoría y retención de estudiantes de educación superior INFORME NACIONAL REPÚBLICA DOMINICANA -.

Himmel, E. (2002). Modelo de análisis de la deserción estudiantil en la educación superior. Calidad En La Educación, (17), 91.

Huesca , M. \& Castaño , M. (2007). Causas de Deserción de Alumnos de Primeros Semestres de una Universidad Privada. (Revista Mexicana de Orientación Educativa), V(12), 34-40.

Jiminián, Y. (2009). Desercion en la Universidad Abierta para Adultos (UAPA), Republica Dominicana.

Martinez, M. (2006). La Investigación Cualitativa ( Síntesis Conceptual ). Revista IIPSI, 9, 123-142.

Rodríguez, A., \& Sánchez, M. (2005). Actualidades investigativas en educacion la deserción estudiantil en la educación superior : el caso de la universidad de costa rica, Costa Rica.

Román, M. (2013). Factores asociados al abandono y la deserción escolar en américa latina: una mirada en conjunto. REICE. Revista Iberoamericana Sobre Calidad, Eficacia y Cambio En Educación, 11(2), 33-59.

Sampieri, R. H., Fernández, C., \& Baptista, P. (2006). Metodología Dela Investigación. Mc Graw Hill.. 